Chapter 4

\title{
Catecholestrogens in Rheumatoid Arthritis (RA): Hidden Role
}

\author{
Wahid Ali Khan and Mohd. Wajid Ali Khan \\ Additional information is available at the end of the chapter \\ http://dx.doi.org/10.5772/51794
}

\section{Introduction}

Sex hormones are implicated in immune response, with estrogens acting as enhancers [10]. Estrogens not only have anti-inflammatory but also pro-inflammatory roles depending upon different influencing factors [66]. Metabolism of estrogen within the body is a complex and important subject. Estrone and estradiol are biochemically interconvertable and yield the same family of estrogen metabolites. The metabolism of estrogen takes place primarily in the liver through Phase I (hydroxylation) and Phase II (methylation, glucuronidation, and sulfation) pathways with ultimate excretion in the urine and feces [26].

Cytochrome P-450 enzymes mediate the hydroxylation of estradiol and estrone, which is the major Phase I metabolic pathway for endogenous estrogens. Several extrahepatic target tissues or cultured cells from target tissue express estrogen-hydroxylating enzymes activities [28]. Each cytochrome P-450 favors the hydroxylation of specific carbons, altogether, these enzymes can hydroxylate virtually all carbons in the molecule, with the exception of the inaccessible carbons. Different functional groups produced by the action of P-450 at the specific sites of steroid nucleus markedly effects the biological properties of different estrogen metabolites. For example, different hydroxylation reaction give estrogenic or carcinogenic metabolites. Functionally, the important reactions catalyzed by cytochrome P-450 are at carbon number 2, 4 and 16 .

In this chapter, we venture into the area of the ambiguous relationship between catecholestrogens (CEs) and rheumatoid arthritis (RA). This analysis is focused in a part on the possible role of CEs in the pathogenesis of RA and on exploring the mechanism behind the generation of autoantibodies taken consideration the role of CEs. It also explains a unique hypothesis, which showed that CEs formed in various tissues undergo oxidative metabolism (enzymatic or non-enzymatic) to produce reactive oxygen species (ROS), which could 
modified DNA and alter it immunogenicity. This would lead to the induction and elevated levels of RA autoantibodies. In addition, chapter further explain the role of CEs in antigendriven induction of RA autoantibodies that may unfold various aspects of RA.

\section{Catecholestrogens (CEs)}

Catecholestrogens (CEs) are biological active metabolites of estrogen which are synthesized by estrogen 2- and 4- hydroxylase in the liver, brain and other organ [29]. They are produced by the actions of genes encoded by CYP1A1, CYP1A2 and CYP1B1, which is an estrogen 4-hydroxylase (Figure 1).

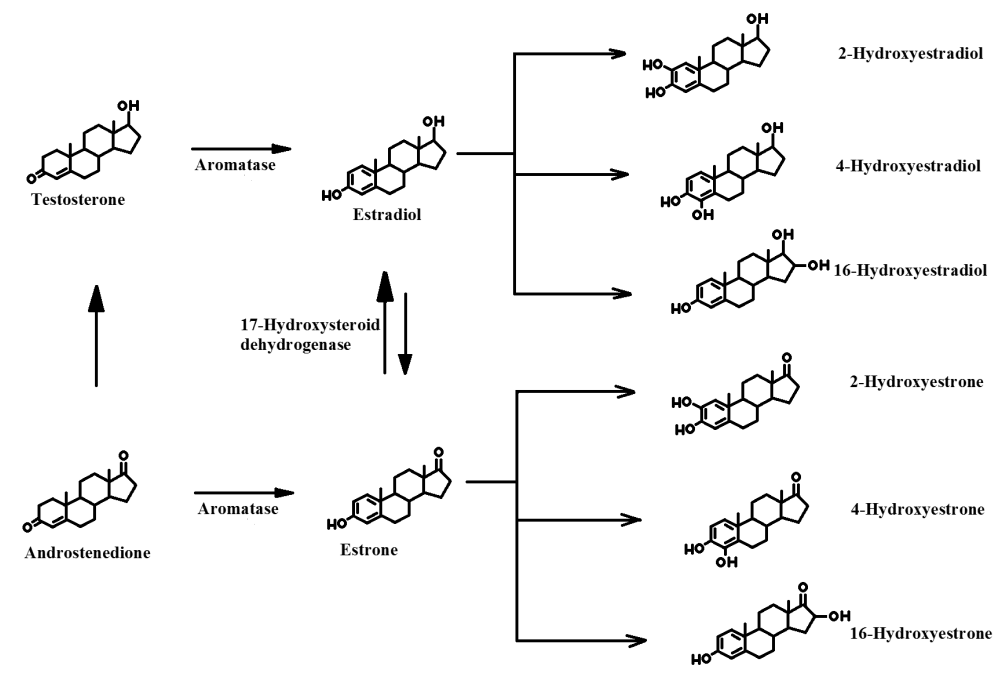

Figure 1. Pathway of estrogen metabolism including estradiol, estrone and various estrogen metabolites (including CEs).

CEs also yield potent genotoxic molecules implicated in carcinogenesis. 4-Hydroxyestrone/ estradiol was found to be carcinogenic in the male Syrian golden hamster kidney tumor model, whereas 2-hydroxylated metabolites were without activity [46]. 4-Hydroxyestrogen can be oxidized to quinone intermediates that react with purine base of DNA, resulting in depurinating adduct that generates highly mutagenic apurinic sites. Quinones derived from 2-hydroxyestrogens produced stable DNA adducts and are presumed to be less genotoxic [67]. As described elsewhere, estrogen may be oxidized by hepatic cytochrome P-450 enzymes to hydroxyl CEs and further oxidized to the semiquinone and quinone form [1]. The quinone formed from oxidation of 4-hydroxyestrogen has half-life of 12 minutes as compared with the short life of 2-hydroxyestrogen $\left(t_{1 / 2}=47 \mathrm{~s}\right)$ [30]. Estrone and estradiol are oxidized to lesser amount to 2-hydroxycatechols by CYP3A4 in liver and by CYP1A in extrahepatic tissues or to 4-hydroxycatechols by CYP1B1 in extra- 
hepatic sites, with the 2-hydroxycatechol being formed to a larger extent. Catechol-o-methyl transferases (COMTs) inactivated 2- and 4-hydroxycatechols to their respective methyl derivatives [69]. COMT, a phase II enzyme, catalyses the transfer of methyl group from S-adenosyl methionine to hydroxyl groups of a number of catechol substrates, including the catecholestrogens. CEs under normal condition are o-methylated by COMT to form 2- and 4-o-methylethers, which are excreted in the urine [56]. Small amount of CEs may also be converted by peroxidase-catalysed reactions to semiquinones or quinones that are capable of forming DNA adducts or generating ROS via redox cycling that could oxidize DNA bases [69]. Estrogen metabolites have indirect and direct genotoxicity. Hydroxylation is an important elimination step for estrogen to generate CEs. Microsomal estradiol hydroxylation in human breast cancer showed significantly higher 4-hydroxy:2-hydroxyestradiol ratio in tumor tissue than in adjacent normal breast tissue [48], but the breast cancer tissue samples contained fourfold higher levels of 4-hydroxyestradiol $\left(4-\mathrm{OHE}_{2}\right)$ than in normal tissue from benign breast biopsies [57].

It has been observed that intra-tissue concentration of estrogens, hydroxyestrogens (16 $\alpha$-hydroxyestrone, 2-hydroxyestrone, 2-hydroxyestradiol, 4-hydroxyestrone, and 4-hydroxyestradiol) and methoxyestrogens (2-methoxyestrone, 2-methoxyestradiol, 4-methoxyestrone, and 4-methoxyestradiol) in normal and malignant breast showed the highest concentration of 4-hydroxyestradiol in malignant tissue [4]. The concentration, as evaluated by combined high performance liquid chromatograpy (HPLC) and gas chromatograpy-mass spectrometry (GC-MS) was found to be more than twice as high as that of any other compound. CEs have been measured in rat brain and various endocrine tissues by sensitive radioenzymatic assay. The concentration of these CEs in the hypothalamus and pituitary are at least ten times higher than parent estrogen. CEs have potent endocrine effect i.e. they have an important role in neuroendocrine regulation [54].

In addition, brain is capable of 2-hydroxylation and consequently forms CEs from estrone and estradiol. Increased availability of estrogen and estradiol for binding and hypothalamic sites would facilitate formation of CEs. 2-Hydroxylated and 4-hydroxylated estrogen exerts biologic responses on the cyclic secretion of leutenising hormone $(\mathrm{LH})$ perhaps even on follicle stimulating hormone (FSH) and prolactin. Catecholestradiol competes with estradiol for estrogen binding sites in the anterior pituitary gland and hypothalamus and dopamine binding sites on anterior pituitary membranes [41].

Mitogenicity associated with estrogen receptor (ER)-mediated cellular events was believed to be the mechanism by which estrogens contributed to carcinogenesis [59]. The role of CEs as genotoxic chemical procarcinogenesis, independent of ER mediation, has been well recognized [70]. Although oxidative metabolism of estrogen to CEs is generally thought to terminate the estrogenic signal, but CEs retain some binding affinity to the ER. Exposure of MCF-7 cells with 2- and 4-hydroxyestradiol increased the rate of cell proliferation and the expression of estrogen-inducible gene such as progesterone receptor (PR) gene and pS2. Compare to estradiol, 2- and 4-hydroxyestradiol increased proliferation rate, level of PR protein as well as pS2 mRNA expression to certain fold [62]. 
As describe previously, estrogens are metabolized via two pathways i.e. formation of CEs and to lesser extent, $16 \alpha$-hydroxylation. The catechols are formed as 2- and 4-hydroxylated estrogen. These two CEs can be inactivated by the enzyme COMT through o-methylation [69]. Other possible mechanism of inactivation includes conjugation of CEs by glucouronidation and sulphation. High concentration of 4-hydroxylated metabolites caused insufficient production of methyl, glucouronide or sulphate conjugate which in turn results in CE toxicity in cell and consequently competitive catalytic oxidation to semiquinone (CE-SQ) and quinone (CE-Q). CE-SQ and CE-Q may conjugate with glutathione (GSH), catalyse by Stransferase. CE-Q may also react with DNA to form stable and de-purinating adduct if this inactivating process is incomplete [47].

\section{CEs and DNA}

Endogenous estrogens can become carcinogenic via formation of catecholestrogen quinones, which react with DNA to form specific estrogen-DNA adducts. The mutations resulting from these adducts can cause cell transformation and initiation of breast cancer [58]. The 4hydroxyestrogen generates free radicals from reductive-oxidative cycling with the corresponding CE-SQ/CE-Q forms, thus causing DNA damage extensively. In comparison to 4hydroxyestrogen, 2-hydroxyestrogen is not carcinogenic and has potent inhibitory effect on the growth of tumor cells and on angiogenesis [17]. The DNA adducts generated by 2-hydroxyestrogen and 4-hydroxyestrogen can form stable modifications that remain in the DNA unless they are removed by repair. On the other hand, the modification bases can be released from DNA by destabilization of the glycosydic bond and result in the formation of depurinated or depyrimidinated sites [7].

Earlier studies from our lab showed that CE modified DNA brings about single and double strand breaks, hyperchromicity, damage at the restriction sites and modification of different bases (Table 1) [40, 36, 37, 38]. It has been observed that CE caused more damage to the DNA in presence of copper in comparison to nitric oxide (NO) as the extent of base modification was greatest for thymine followed by guanine, adenine and cytosine [40]. We proposed that $\mathrm{CE}$ and NO formed in different tissue may react with each other to produce CE-Q.

The quinone/semiquinone redox system produces superoxide ions $\left(\mathrm{O}_{2}^{-}\right)$which can react with NO to form peroxynitrite, which could cause DNA damage [38]. Quenching studies of DNA modified with CEs in presence of C(II) showed that catalase and bathocuproine strongly ( $80 \%$ ) inhibited the modification by CEs plus $\mathrm{Cu}(\mathrm{II})$ indicating the involvement of $\mathrm{H}_{2} \mathrm{O}_{2}$ and $\mathrm{Cu}(\mathrm{I})$ during the modification $[36,37]$. These results demonstrate that CEs lead to the production of potent ROS, capable of causing DNA damage, thus playing important role not only in carcinogenesis [36] but also in systemic lupus erythematosus (SLE) [40, 37, 39] and RA [38]. 


\begin{tabular}{|c|c|c|}
\hline Parameter & Native DNA & CE-modified DNA \\
\hline Hyperchromicity & - & 32.2 \\
\hline Absorbance ratio $\left(\mathrm{A}_{260} / \mathrm{A}_{280}\right)$ & 1.86 & 1.52 \\
\hline Melting temperature $\left(\mathrm{T}_{\mathrm{m}}\right)^{\circ} \mathrm{C}$ & 88 & 80.5 \\
\hline S1 nuclease action & No digestion & Digestion \\
\hline \multicolumn{3}{|c|}{ Restriction enzymes } \\
\hline 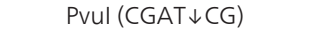 & Digestion & No digestion \\
\hline Pvull (CAG $\downarrow C T G)$ & Digestion & No digestion \\
\hline Sspl (AAT $\downarrow A T T)$ & Digestion & Digestion \\
\hline \multicolumn{3}{|c|}{ Circular dichroism (mdeg) Wavelength ( $\mathrm{nm}$ ) } \\
\hline+274 & 0.77 & 0.52 \\
\hline-246 & -1.3 & -7.60 \\
\hline-210 & -0.85 & -0.58 \\
\hline Scavengers & & of modification \\
\hline Catalase & - & 82 \\
\hline Sodium azide & - & 21 \\
\hline Bathocuproine & - & 80 \\
\hline Ascorbic acid & - & 35 \\
\hline \multicolumn{3}{|c|}{ Base modification } \\
\hline Adenine & - & $16.9 \%$ \\
\hline Guanine & - & $45.5 \%$ \\
\hline Cytosine & - & $12.6 \%$ \\
\hline Thymine & - & $47.1 \%$ \\
\hline
\end{tabular}

Table 1. Characterization of Native and CE-modified DNA under identical experimental conditions. (Adapted from [36]).

Over the last 2 decades, only two studies have been reported that explain the presence of $\mathrm{CE}$ adducts in human breast tissue. First study involves the analysis of CE adducts in the DNA from malignant breast tumor. DNA sample showed the presence of deoxyguanosine adducts of 4-hydroxyestradiol and 4-hydroxyestrone in almost every samples [21]. Other study showed the presence of CE quinone-derived DNA adduct in two breast samples that were collected from one women with and one woman without breast cancer [51]. The catechol quinone-derived adducts identified were 4-hydroxyestradiol-1-N3-adenosine, 4-hydroxyestrone-1-N3-adenosine and 4-hydroxyestradiol-1-N7-guanine. Animal experimentation involves the injection of 4-hydroxyestradiol (or estradiol-3,4-quinone) into the mammary glands of female ACI rats which resulted in the formation of the depurinating adducts, 4- 
hydroxyestradiol-1-N3-adenosine and 4-hydroxyestradiol-1-N7-guanine. [47]. In addition to these adducts, 4-hydroxyestradiol-GSH conjugates was also detected. Another study demonstrates the presence of 4-hydroxy catecholestrogen conjugates with GSH or its hydrolytic product (cysteine and $\mathrm{N}$-acetylcysteine) both in tumors and hyperplastic mammary tissues in ERKO/Wnt-1 mice [18]. Estradiol-3,4-quinone reacted rapidly to form 4-hydroxyestradiol-1-N3-adenosine adducts that are considered to be as depurinating adducts.

It has been shown that CEs can induce DNA lesions not only in plasmid DNA [40, 36, 37, 38] but also in calf thymus DNA [49]. In short, quinone intermediates produced by oxidation of the CE 4-hydroxyestradiol or 4-hydroxyestrone may react with purine bases of DNA to form depurinating adducts that generate highly mutagenic apurinic sites. In contrast, quinone of 2-hydroxylated estrogen produce less harmful and stable DNA adducts [7]. The CE metabolites may also produced potentially mutagenic oxygen radicals by metabolic redox cycling that can also caused DNA damage. The abasic sites resulting from the spontaneous depurination-depyrimidination of the modified bases and the aldehydic base and sugar lesions resulting from the oxidative damage to deoxyribose moieties in the DNA molecules [49], disturb the structural integrity of DNA and destabilize the double helix. This would alter it property and render DNA immunogenic.

\section{CEs and Immune Response}

Despite a large number of reports that may explain the role of estrogen (or estradiol) in modulation of immune response [10,66], the role of CEs in the immune response is lacking. It has been observed that the conversion from 2-hydroxysetrogens to 4-hydroxyestrogen might be an additional pro-inflammatory signal because these endogenous metabolites can be oxidized to 3,4-quinone, which lead to depurination and mutation [49]. The 3,4-semiquinone and quinone produce ROS, which stimulate inflammation, whereas methylation and sulfation are detoxification pathways, which involve the release of detoxying agents in the urine [56]. Other pro-inflammatory role of 4- hydroxyestrogen can be explain by the fact that 4-hydroxyestradiol undergoes 2-electron oxidation to quinone via semiquinone and during this process, ROS can be generated to cause DNA damage and cell death [8]. A similar effect was observed in our studies that explained extensive DNA damage and base modification [40, 36, 37, 38, 39]. In contrast to the pro-oxidant activities of 4-hydroxyestradiol, 2-hydroxyestradiol is a antioxidant that show no carcinogenic activities and has potent inhibitory effect on the growth of tumor cells and on angiogenesis [17]. Thus, 4-hydroxyestradiol in comparison to 2-hydroxyestradiol induce carcinogenesis and have pro-inflammatory effects [20]. But now, we are unaware of the fact that whether pro-inflammatory mediators induce a shift from 2-hydroxyestrogen to 4- and 16-hydroxylated estrogens. From all these studies there is a clear evidence that 2-methoxyestradiol is a pro-apoptotic and cytostatic endogenous compound, which can inhibit not only angiogenesis [17] but also inflammation in animal models. In contrast, 4-hydroxylated estrogens might exert pro-inflammatory roles by inducing ROS and DNA damage. Just like conversion from 2-hydroxyestrogens to 4-hydrox- 
yestrogens, a shift to $16 \alpha$-hydroxylated forms of estrogen can be an important pro-inflammatory and pro-proliferative signal [66].

The direct role of CEs in mediating immune response can be explain in our studies that demonstrate the production of antibodies to CE-modified plasmid DNA [40, 36, 37, 39, 38]. As reported earlier, CE-modified DNA (CE-DNA) was found to be highly immunogenic (> 1:12800) in animal model [36]. These antibodies were effectively used as probe for detecting oxidative lesions in human genomic DNA as well as for the estimation of 8-hydroxy-2'-deoxyguanosine (8-OHG) level in the urine of cancer patients [36] and RA patients [38]. Antibodies evaluated from competition ELISA showed strong specificity for the CE-DNA (immunogen) causing about $92 \%$ inhibition in anibody binding at $20 \mu \mathrm{g} / \mathrm{ml}$ of the immunogen (competitor) concentration. Fifty percent inhibition was observed at $3.5 \mu \mathrm{g} / \mathrm{ml}$ of the immunogen. In addition, these antibodies showed a very high degree of binding toward 8OHG that may explain the role of ROS in mediating various immune responses including inflammation. CE-modified form of different bases (adenine, guanine, cytosine, thymine), human and calf thymus DNA, ROS-DNA, poly nucleotides (dT, dA, dC, dG, dA-dT) were also recognized by these antibodies. Another evidence that explain the role of CEs in mediating immune responses comes from our previous study that showed that CE-DNA was not only recognized highly by cancer autoantibodies [36] but also by anti-DNA autoantibodies in SLE [40,37] and RA [38]. Results from all these studies demonstrate that CEs somehow produce some antigenic stimulus that may contribute to the generation of antibodies which may directly or indirectly affect the immune system.

Dual pro-inflammatory and anti-inflammatory role of estrogens has been described earlier [66], which depend on the immune stimulus, subsequent antigen-specific immune response, the cell types involved during different disease phase, the specific microenvironment, timing of estrogen administration in relation to the disease course, the concentration of estrogen, the variability in expression of estrogen receptor depending on the microenvironment and the cell type and intracellular metabolism of estrogen leading to important biologically active metabolites (CEs) with quite different anti- and pro-inflammatory function. In addition, one of the striking property of estrogen is its ability to differentiate $\mathrm{T}$ and $\mathrm{B}$ cells, increase immunoglobulin production and increase immune complex-mediated disease such as SLE [9]. It has been found that women, who are diagnosed with breast cancer, have significantly elevated anti-HMdU autoantibodies [23]. This study is in agreement with our previous finding that explains the presence of circulating antibodies against CE-modified DNA in cancer patients [36]. The modified DNA presents unique epitopes which may be one of the factors for autoantibody induction in cancer. The presence of high levels of these antibodies proves to be the pro-oxidant conditions that have led to the oxidation of bases in genomic DNA and have stimulate an immune response. Since native DNA is a known weak immunogen, it appear that DNA damage by CE render it immunogenic leading to the induction of cancer autoantibodies. It has been suggested that the oxidative DNA damage and the immunologic responses it evokes (autoantibodies) start occurring not only in the carcinogenic process [36] but also in various autoimmune diseases [40, 36, 37, 39, 38]. Estrogen also modulate the function of PMNs (poly morphonuclear leukocytes, neutrophils, granulocytes), 
which in turn cause the production of ROS on their stimulation. ROS generated by PMNs cause DNA modification as well as oxidation of protein and lipid peroxidation. It is interesting to note that 2-hydroxylated estrogens works as powerful inhibitors of PMNs activity, which shows one of the protective property of the 2-hydroxylated CEs. Therefore, estrogens (and estrogen metabolites like CEs) affect immunological responses and in turn, their activities are affected by the immunological products. In addition, estrogen in physiological concentration serves to enhance immune response by inducing the production of various types of cytokines and interleukins.

\section{CEs and Cytokines}

The sex hormones are likely to directly modulate the function of cells involved in the immune response [42]. The pro- and anti-inflammatory effects of estrogen and their metabolites (CEs) on secretion of pro-inflammatory cytokines have been a matter of debate for two or three decades. In fact, at physiological dose, estradiol induce IL-1 $\alpha$, a cytokine that can initiate a cascade of other cytokines, chemotactic and growth factors [12]. It is interesting to note that estradiol also inhibits IL- $1 \alpha$-induced IL-6 production. Therefore, estradiol not only results in increased human epithelial cell proliferation (a process important in tumor growth) but also inhibits the activity of natural killer cells, thus allowing tumor growth [52].

Estrogen and their metabolites (such as CEs) stabilized or increased cytokine secretion whereas testosterone inhibited this secretion [32]. This study explains that down stream estrogen, 2-hydroxyestradiol (2- $\left.\mathrm{OHE}_{2}\right), 4-\mathrm{OHE}_{2}$ and 16-hydroxyestradiol $\left(16-\mathrm{OHE}_{2}\right)$ did not stimulate TNF secretion. However, the combination of $16-\mathrm{OHE}_{2}$ and $2-\mathrm{OHE}_{2}$ or $4-\mathrm{OHE}_{2}$ markedly stimulated TNF secretion that was observed in presence of cortisol. Therefore, at physiological concentration, estradiol and a combination of downstream estrogens (like 2$\mathrm{OHE}_{2}, 4-\mathrm{OHE}_{2}$ and 16- $\mathrm{OHE}_{2}$ ) increased (or stabilized) immune stimuli-induced TNF secretion. In addition, estradiol also caused the production of IL-6, IL-2, IFN $\gamma$, IL-4 and IL-10, caused by the pro-inflammatory influence of applied immune stimuli [32]. The study of Janele et al, 2006.] demonstrates that ratio of 16-hydroxylated estrogen in relation to 2-/4-hydroxylated estrogen is important for TNF secretion. It has been found that the ratio of 10:1 of $16-\mathrm{OHE}_{2}$ in relation to 2-/4- hydroxylated estrogen markedly stimulated TNF secretion in presence of cortisol (Figure 2).

In addition, in absence of cortisol, the combination of 16-hydroxylated and 2-hydroxylated estrogens even strongly inhibited TNF secretion. During chronic inflammatic conditions, administration of therapeutic estrogens increased 16-hydroxylated estrogens. In addition, during chronic inflammation in the situation of rheumatic diseases (example RA) the balance is switch to estrogen and particularly to 16-hydroxylated estrogen. In conclusion, one should assume that therapeutic administration of estrogen would enhance $16-\mathrm{OHE}_{2}$ over 2-/4-hydroxylated estrogens, which would support secretion of pro-inflammatory cytokines. 


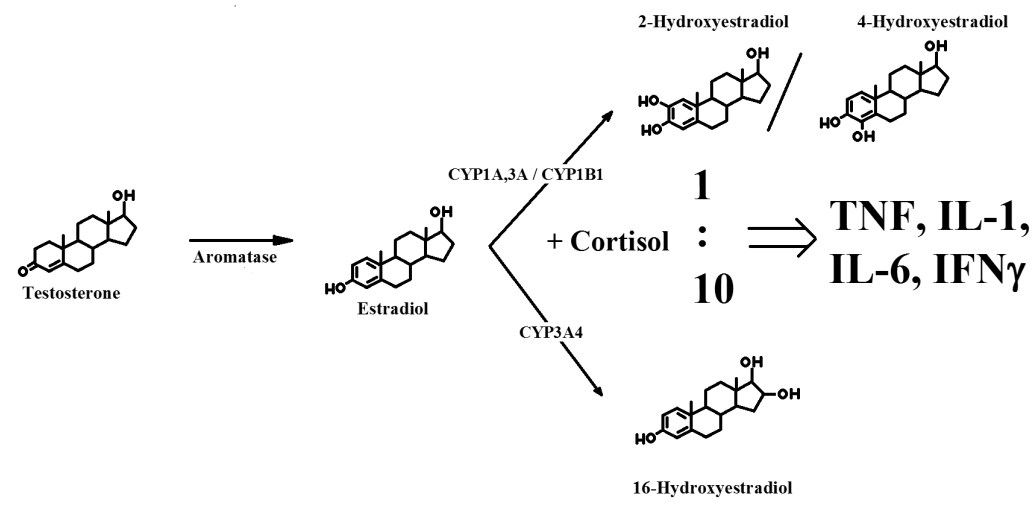

Figure 2. Effect of estrogen and their catechol metabolites (CES) on the secretion of pro-inflammatory cytokines in RA.

It has been observed that estradiol increased IgG and $\operatorname{IgM}$ production by peripheral blood mononuclear cells (PBMC) in patients of SLE. This led to elevated levels of polyclonal IgG (including IgG anti-dsDNA) by enhancing B-cell activity by way of IL-10 [33]. It should be important to check these results in the presence of $16 \alpha$-hydroxyestrone $\left(16-\mathrm{OHE}_{1}\right)$ and the naturally occurring 2-hydroxylated CE. Previous study shown that decrease activity in SLE patients correlated negatively with urinary concentration of 2-hydroxylated estrogens [68]. Interestingly, estradiol increase IL-10 production by monocytes and exogenous IL-10 further enhances estradiol-induced increase in antibody production by B cells. Therefore, in SLE patients, estradiol increases IL-10 production by patient's monocytes and exogenous IL-10 acts additively with estradiol to increase antibody production of B cells. It is hypothesized that estradiol-estradiol receptor complexes may enhance the transcription of IL-10 genes by binding to estrogen-responsive elements or by binding to some transcription factor that directly bind those elements [24].

High doses of estrogen (1500-2000 pg/ml) administration to non-overiectomized mice ameliorated disease systems and significantly decreased the percentage of TNF $\alpha$ or IFN $\gamma$ producing CNS antigen-specific T Cells in both the CNS and spleen [31]. However, similar studies in mice lacking IFN $\gamma$, IL-4 and IL-10 genes provide evidence that these cytokines may not be necessary for estradiol-mediated improvement of disease severity, discrediting the notion that an estrogen-induced shift towards Th 2 cytokine involved [31]. Estrogen also stimulates secretion of IL-4, $-5,-6$ and -10 by $\mathrm{T}_{\mathrm{H}} 2$ lymphocytes. These cytokines are potent stimulators of B-cell proliferation, maturation into plasma cells and synthesis of antibody. Interleukins 4, 5, 6 and 10 are expressed in greater amount in an estrogen-dominant hormonal milieu [53]. SLE patients have increased level of IL- 6 and IL-10, product of $\mathrm{T}_{\mathrm{H}} 2$ lymphocytes and macrophages, which can be directly correlated with clinical disease severity [53]. Patients with lupus also have an inherent defect in interferon gamma production. Under normal condition, increased estrogen leads to an increase interferon gamma concentration but in SLE patients, the estrogen-associated increase in interferon gamma secretion is absent 
[53]. In addition, interesting changes of serum estrogens that correlated with cytokine variation have been found during pregnancy in SLE patients [19]. The local effect of sex hormones in autoimmune rheumatoid disease (RA) seem to consist mainly of altered cell proliferation (i.e. estrogen enhance) and cytokine production.

\section{Reminding the concept of rheumatoid arthritis (RA)}

Rheumatoid arthritis (RA) is an autoimmune disease whose aetiology and progression are multifactorial, including a range of immune, neuroendocrine and psychosocial variable [14]. How these variable interact with one another and how they ultimately influence the disease process in RA is only partially know. A growing body of evidence showed that the stress system and its interaction with the immune system, play a vital role in RA [14]. RA is more prevalent among women than men and $80 \%$ of the total cases occurring between the age of 35 and 50 [34]. The primary presenting symptoms are pain, stiffness and swelling of the joints resulting in impaired physical function. Synovial inflammation underlines the cardinal manifestations of this disease, which include pain, swelling and tenderness followed by cartilage destruction, bone erosion and subsequent joint deformities. In RA, joint involvement is typically symmetric, a characteristic usually not found in other forms of arthritis [50]. Early theories on the pathogenesis of RA focused on autoantibodies and immune complexes, $\mathrm{T}$ cell-mediated antigen-specific responses, $\mathrm{T}$ cell-independent cytokine network and aggressive tumorlike behavior of synovium have also been implicated [22]. Recently the contribution of autoantibodies in RA has come in the front line and specific therapeutic interventions can be designed to suppress synovial inflammation and join destruction in RA [22].

RFs have been the hallmark autoantibody found in RA [71]. Autoantibodies to the major cartilaginous collagen have been found in the sera of some RA patients. In addition, cross-reactive natural autoantibodies (IgM) have been described in the sera of RA patients [25]. These antibodies are predominantly directed against histone moieties or against double-stranded DNA. Anti-ds DNA has also been reported following treatment of RA with IFN $\gamma$. Beside that, autoantibodies have been found in the sera of RA patients that showed preferentially high binding of CE-modified DNA [38]. In addition, the antibodies have been shown to represent a alternative immunochemical probe to detect oxidative lesions in DNA (genomic) as well as for the estimation of 8-OHdG level in different body fluid of RA patients [38], which may be used as marker in the diagnosis of the disease. Many autoantibody system that could participate in inflammatory joint disease are now recognized in RA, including rheumatoid factor, type II collagen, immunoglobulin heavy gene binding protein, heat shock proteins and hn RNP-33 [64]. Anti-collagen antibody and rheumatoid factor are produced by rheumatoid synovial B cells. The ability of ubiquitous antigen (glucose-6-phosphoisomerase) to induce synovial inflammation is probably related to their adherence to the cartilage surfaces. The presentation of immobilized antigen-antibody complexes on cartilage provides an exceptionally good substrate for complement fixation, similar to rheumatoid factor embedded in rheumatoid cartilage. 
Macrophage-derived cytokines (like tumor necrosis factor alpha, TNF $\alpha$ ) know to play an important role in the induction and perpetuation of the chronic inflammatory processes in rheumatoid joints as well as in the systemic manifestation of this disease [27]. Over production of this cytokines in the joints of RA patients triggers increase in synoviocyte proliferation and a cascade of secondary mediators involved in the recruitment of inflammatory cells and in the process of joint destruction [3]. It has been found that increase inflammatory markers are responsible for disease progression and joint destruction in early RA. Infect the rate of cartilage and joint destruction is correlated with plasma elevation in inflammatory acute phase reactants (C-reactive protein and vascular endothelial growth factor) and in the synovial concentration of matrix metalloproteinase, matrix digesting enzymes directly responsible for joint damage [2].

In recent years the treatment of RA has undergone somewhat of a revolution, with a strong consensus emerging in favor of early, aggressive therapy [63]. There is growing evidence that early treatment of the disease has a beneficial impact on treatment outcome. The goal to be achieved in managing RA are to explain the underline mechanism behind the generation of antibodies to cartilaginous collagen as well as other oxidative stress condition generated in the synovial fluid, which may be used as marker in the early diagnosis of the disease.

\section{Role of CEs in the aetiopathogenesis of RA}

Estrogen and their CE metabolites know to play an important role in autoimmune rheumatic diseases $[38,68,15]$. A role for estrogen in the pathogenesis of RA has been review for the last few years $[66,70,38,12,32,68,15]$ but the exact patho-aetiology remains elusive. The evidences concerning the possibility of CEs in the development of RA are very limited and preliminary. It has been observed that the ratio of $16-\mathrm{OHE}_{1} / 4-\mathrm{OHE}_{2}$ in synovial fluid (SF) of RA was found to be significantly higher than control [6]. In addition, SF level of 4-OHE $\mathrm{OH}_{2}$ was significantly increased in RA patients compared to controls [5]. Interestingly, urinary concentration of 2-hydroxylated estrogen were 10 times lower in patients with RA than in healthy individuals but the ratio of $16-\mathrm{OHE}_{1} / 2$-hydroxylated was 20 times in RA patients as compared to control [68]. These finding suggests that the magnitude of conversion to the mitogenic $16-\mathrm{OHE}_{1}$ is extremely upregulated in RA which most likely contributes to the maintenance of the disease. Furthermore, peripheral estrogen hydroxylation was found to be increased in both men and women with SLE and estrogenic metabolites were reported to increase B cell differentiation and activate T cells [44].

Both RA and SLE are associated with an altered sex hormone balance characterized by lower amount of immunosuppressive androgens and higher amount of immune enhancing estrogens [15]. Increased estrogen to androgen ratios have been observed in RA patient's SF because of increased aromatase expression by inflammatory macrophages infiltrating synovial tissue [5]. Increased estrogen concentration in RA SF from patients of both sex likely results from the pro-inflammatory cytokines TNF $\alpha$, IL- $1 \beta$ and IL- 6 , which accelerate the metabolic conversion of estrogen from androgen by inducing the synovial tissue aroma- 
tase (Figure 3). As a result, increased estrogen level might exert activating effects on synovial cell proliferation including macrophages and fibroblast [35]. Interestingly, renal excretion studies showed that the enhanced estrogen metabolism observed in both RA and SLE patients resulted in elevation of pro-inflammatory metabolites derived from estrone or estradiol such as $16-\mathrm{OHE}_{1}$, which exert mitogenic effects and may induce proliferation of synovial fibroblast $[68,13]$. In addition, RA SF showed reduced amounts of anti-inflammatory estrogens metabolites such as 2-hydroxyestrogen that inhibits the growth promoting effects of estrogen. In contrast, $16-\mathrm{OHE}_{1}$ metabolites considered as enhancer of cell proliferation. In RA synovial tissue, biological effects of estrogen metabolites as a consequence of altered peripheral sex hormone synthesis mainly results in stimulation of cell proliferation and cytokine production [11].

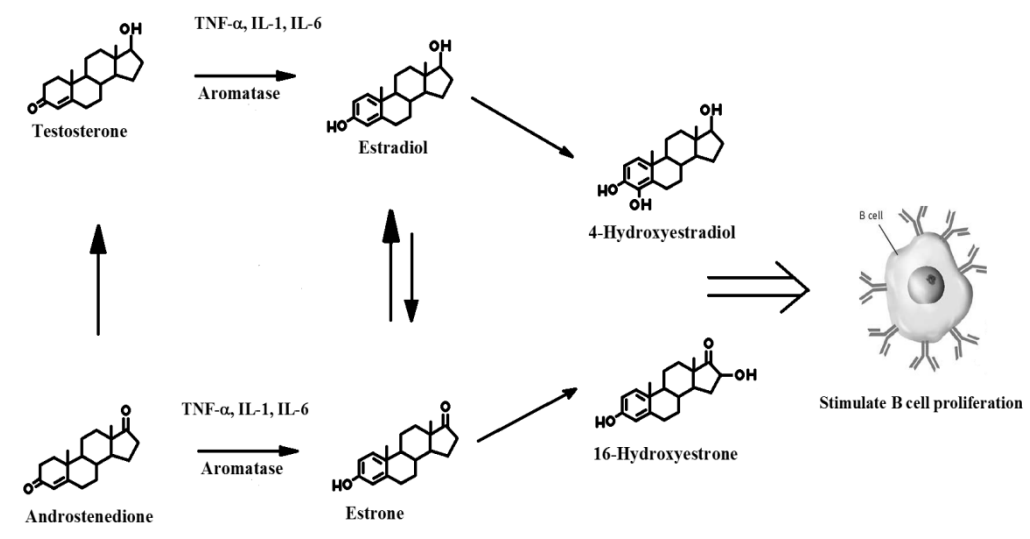

Figure 3. Immune modulation by estrogen and their metabolites in the synovial tissue of RA patients.

It has been observed that RA synovial cells mainly produce the cell pro-proliferation 16$\mathrm{OHE}_{1}$, which in addition to $16-\mathrm{OHE}_{2}$, is the downstream estrogen metabolites that interferes with monocyte proliferation. In addition, urinary concentration and total urinary loss of 2-hydroxyestrogen was found to be 10 times higher in healthy subjects compared to RA [11]. Peripheral metabolic conversion of androgens to potent metabolites of estrogen that promote immune cell proliferation and activation may have role in the female predisposition to autoimmunity.

Estrogen represent a risk factor for the development of autoimmunity and therefore, their therapeutic use must be avoided in patients with active immune-mediated diseases specially RA [16]. In addition to estrogen, various CE metabolites know to increase the risk for the development of RA disease. The increased estrogen concentration has been observed in RA $\mathrm{SF}$, where the hydroxylated forms make the major concentration in particular $16-\mathrm{OHE}_{1}$ and $4-\mathrm{OHE}_{2}$ whereas, the 2-OHE $\mathrm{H}_{1}$ was found to be similar to the controls [6, 5]. As describe early, $16-\mathrm{OHE}_{1}$ is a mitogenic and proliferative endogenous hormone which is responsible for the 
proliferation of synovial fibroblast [13]. 16- $\mathrm{OHE}_{1}$ is formed from upstream estrone and estradiol, which show biological responses by covalent linkage to the receptor [61]. The other conversion product of estrone and estradiol are the 2-hydroxylated estrogens such as 2$\mathrm{OHE}_{2}$ and $2-\mathrm{OHE}_{1}$, which would classified as CE. In comparison to 16 hydroxylated estrogens, the 2-hydroxylated forms of estrogen inhibit growth-promoting effects of estradiol [61]. In one of the previous study, it has been observed that urinary levels of 2-hydroxylated estrogens were found 10 times lower in RA patients in comparison to normal subjects, whereas the ratio $16-\mathrm{OHE}_{1} / 2$-hydroxyestrogens was found to be 20 times higher in RA patients than in healthy controls [68]. Decreased loss of 2-hydroxylated estrogen in relation to the mitogenic $16-\mathrm{OHE}_{1}$ might provide important stimulus to support proliferative state of the synovial cells in RA. Therefore, concentrated-related conversion to pro- and anti-inflammatory down stream metabolites of estrogen might support dual role of estrogen (pro- or anti-inflammatory) [13].

Estrogen at physiological concentration serves to enhance immune responses that may act as important driving force for human humoral immunity [65]. Estradiol increase IL-10 production by monocytes and exogenous IL-10 further enhance estradiol-induced increase in antibody production by B cells. In addition, estradiol enhances IgG and IgM production by PBMC, which leads to elevated levels of polyclonal IgG, including IgG anti-DNA in PBMC of SLE patients by enhancing B cell activity via interleukin 10 [33]. These studies are in agreement with our previous studies that describe the role of CEs in the etiopathogenesis of SLE, cancer and RA [40, 36, 37, 38, 39]. To have a better insight into the possible role of CEs in the aetiopathogenesis of RA, we have demonstrated that CE-modified DNA was highly recognized not only by SLE IgG [40, 37, 39] and cancer IgG [36] but also by RA IgG [38], pointing out the possible participation of modified DNA in the pathogenesis of various autoimmune diseases including RA, as it has been reported that CE-modified DNA bases cause DNA strand breakage and adduct formation in vivo and in vitro [7]. CE-modified DNA showed preferentially high binding with RA sera when compare with nDNA ( $\mathrm{p}<$ 0.001). These studies clearly indicate that the modified DNA is an effective inhibitor showing substantial difference in the recognition of CE-modified DNA over the native form [38]. The results also demonstrate the pro-oxidant condition which is generated due to the oxidative metabolism of estrogen [40,36]. Non-enzymatic oxidation of CE in the presence of NO [40] or $\mathrm{Cu}$ (II) [37] cause DNA modification and enhanced binding of these modified antigens to RA autoantibodies. To further confirm the role of CEs in RA, we evaluated the binding of autoantibodies by quantitive precipitin titration. The apparent association constant clearly indicates better recognition of CE-modified DNA over native by RA autoantibodies. The enhanced recognition of CE-modified DNA by RA IgG indicates possible participation of modified DNA in RA pathogenesis. The spontaneous production of autoantibodies in RA might arise as a consequence of antigenic change in DNA. Therefore, it could be possible that CE-modified bases of DNA might be one of the contributing factors towards the production of autoantibodies. However, the pro-inflammatory role of estrogen cannot be ruled out [65]. It might be possible that estrogen caused $\mathrm{T}$ and $\mathrm{B}$ cell differentiation and increase immunoglobulin production. These autoantibodies might be strongly bound with CE-modified DNA than native polymer. Further more, DNA isolated from sera and SF of different 
RA patients caused appreciable inhibition in the activity of anti-CE-modified DNA antibodies clearly showing the presence of oxidatively altered epitopes on the isolated DNA molecules, which could be directly correlated to estrogen/radical mediated oxidative stress [38]. These studies show that hydroxylated estrogens (specially CEs) might have a role in the aetiopathogenesis of RA and other autoimmune diseases.

Various CE metabolites $\left(4-\mathrm{OHE}_{2}, 4-\mathrm{OHE}_{1}, 2-\mathrm{OHE}_{2}, 2-\mathrm{OHE}_{1}\right)$ known to secret various cytokines from human peripheral blood leukocytes in presence of cortisol [32]. It has been demonstrated that the ratio of 16-hydroxylated estrogen in relation to 2-/4-hydroxylated estrogen is important for TNF secretion. The study showed that the ratio of 10:1 of $16-\mathrm{OHE}_{2}$ in relation to 2-/4-hydroxylated estrogens stimulate TNF secretion in the presence of cortisol otherwise this effect was not observed in absence of this hormone. Furthermore, absence of cortisol strongly inhibited TNF secretion if the combination of $16-\mathrm{OHE}_{1}$ and 2-hydroxylated estrogens were used in this experiment [32]. In addition, it has been demonstrated that estrogens are able to enhance secretion of matrix metalloproteinase and IL- $1 \beta$-induced IL- 6 secretion from human fibroblast-like synoviocytes in RA [35]. However, with respect to serum levels of estrogen in RA patients, they are not changed which is in strict contrast to androgen levels [13]. High estrogen concentration has been found in particular in SF of RA patients. The presence in the RA SF of an altered sex hormone balance resulting in lower immunosuppressive androgen and higher immunoenhancing estrogens might determine a favourable condition for the development of the immune-mediated RA synovitis. The appropriated explanation for high concentration of estrogen (and their CE metabolites) can be originated from the study that showed that the inflammatory cytokines (TNF- $\alpha$, IL-1, IL-6) are particularly increased in RA synovitis and stimulate aromatase activity in peripheral tissues [55]. Therefore, enzyme complex is responsible for the peripheral conversion of androgen to estrogen (estrone and estradiol). In addition, a significant correlation was found between the aromatase activity and IL-6 production and aromatase has also been found significantly in synoviocytes [45]. Therefore, the increased aromatase activity induced by locally produced inflammatory cytokines (TNF- $\alpha$, IL-1, IL-6) might explain the altered balance resulting in lower androgens and higher estrogen in RA SF as well as their effects on synovial cells $[15,6]$. In conclusion, an increase in 16-hydroxyestrogen relative to the sum of all 2and 4-hydroxylated estrogens (CE) must be viewed as a pro-inflammatory signal, which is particularly evident in RA patients.

Elevated serum concentration of $16-\mathrm{OHE}_{1}$ have been described in patients with SLE [43], indicating that abnormal pattern of estradiol metabolism may lead to increased estrogenic activity. Interestingly, similar phenomenon was observed in the synovial fluid of RA patients, where $16-\mathrm{OHE}_{1} / 4-\mathrm{OHE}_{2}$ was found to be significantly higher compared with control fluid [6, 5]. 4-Hydroxylated estrogens were found to be mitogenic and thus have pro-inflammatory effect $[66,68]$. Importantly, all 2- and 4-hydroxylated estrogen demonstrated TNF-inhibitory effect, which was not observed with $16 \alpha$-hydroxylated estrogen. Thus the observed preference of $16 \alpha$-hydroxylated over 2-/4-hydroxylated estrogens must be considered an important pro-inflammatory signal, which occur particularly in RA patients. This study also describes methylation of hydroxylated estrogen metabolites (CEs) in addition to hydroxyla- 
tion of estrone and estradiol. Catechol-o-methyl transferase, the only enzymes responsible for the methylation of CEs, was also present in primary synovial cells, although it doesn't show any preference with either estrone or estradiol-hydroxylated substrate. Similar to the 2- and 4-hydroxylated forms of estrogen, the 2- and 4-methoxy form inhibited TNF secretion from RA and osteoarthritis (OA) synovial cells [60]. These studies show that hydroxylated estrogen (especially CEs) might have a role in the aetiopathogenesis of RA and other autoimmune diseases.

\section{Conclusion}

Remarkable progress have been made in understanding the role of estrogen in the etiology of RA but the role of CEs in RA is lacking. Catechol metabolites known to play an important role in RA but the exact patho-aetiology remain elusive. The evidence concerning the possibility of CEs in the development of RA is very limited and preliminary. It has been observed that oxidative reactions, often catalyzed by isoforms of the cytochrome P-450, can result in the formation of CEs from parent estrogen and subsequently, semiquinones and quinones derived from CEs, are capable of forming either stable or depurinating DNA adducts. Oxidation of CEs also leads to high amount of ROS that can generate extensive DNA damage. This would probably alter its immunogenicity leading to the induction and elevated levels of RA autoantibodies (Figure 4). Therefore, it is possible that the CE-modified bases of DNA might be one of the contributing factors towards production of SLE autoantibodies. In addition, estrogen not only induces DNA damage but also modulate immune response and immune mediated disease. Estrogen was found to increase IgG and IgM from PBMC, which let to elevated level of polyclonal IgG including IgG anti-ds DNA by enhancing B cell activity via IL-10. These autoantibodies could be strongly bound to DNA and serve as an immunological marker for the diagnosis of diseases. Estrogen is thought to play both pro- and antiinflammatory role in chronic inflammatory diseases that were found to be related to low and high concentration. Increased estrogen to androgen ratio have been observed in RA patients SF, perhaps due to increase aromatase expression by inflammatory macrophages infiltrating synovial tissues. The discovery of high concentration in SF from RA patients of both sexes can also be explain by the fact that inflammatory cytokines (i.e. TNF $\alpha$, IL-1, IL-6) are increased in RA cynovitis and can markedly stimulate aromatase activity in peripheral tissue. But renal excretion studies showed that enhanced estrogen metabolism observed in RA resulted in elevation of pro-inflammatory metabolites derived from estrone and estradiol such as $16-\mathrm{OHE}_{1}$ (or $4-\mathrm{OHE}_{2}, 4-\mathrm{OHE}_{1}$ ) which exert mitogenic effect on different synovial fibroblast. In contrast, RA SF have reduced amount of anti-inflammatory estrogen metabolites such as 2-hydroxyestrogen that inhibit the growth-promoting effect of estradiol. An unwanted shift from 2- to 4-hydroxyestrogen might be an additional pro-inflammatory signal because these 4-hydroxylated metabolites can be converted to 3,4-quinone, which lead to depurination and mutation in DNA. In conclusion, an increase in $16-\mathrm{OHE}_{1}$ relative to the sum of all 2- and 4-hydroxylated estrogens must be considered as pro-inflammatory signal which is particularly evident in RA [60]. 


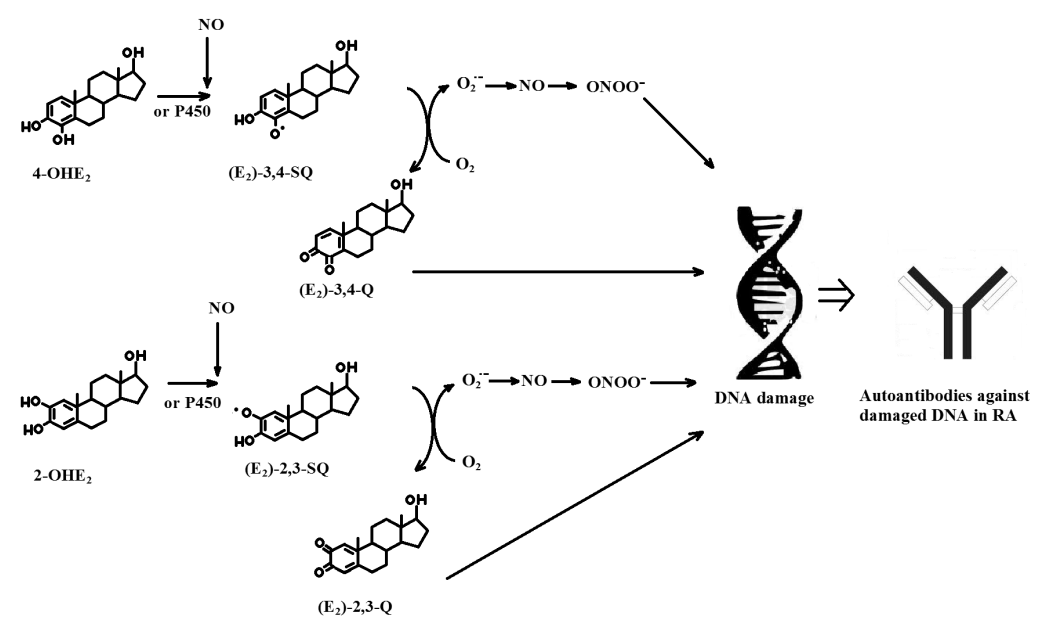

Figure 4. The proposed mechanism for the production autoantibodies in $\mathrm{RA}$. 4- $\mathrm{OHE}_{2}$ (4-hydroxyestradiol), 2-OHE 2 (2hydroxyestradiol), ( $\left.E_{2}\right)-3,4-S Q$ (Estradiol-3,4-semiquinone), ( $\left.E_{2}\right)-2,3-S Q$ (Estradiol-2,3-semiquinone), ( $\left.E_{2}\right)-3,4-Q$ (Estradiol-3,4-quinone), ( $\left.E_{2}\right)-3,4-Q$ (Estradiol-2,3-quinone).

In light of these data, the review literature leads to the conclusion that CEs (and other metabolites) might have a role in the pathogenesis of RA. Based on the preliminary report, it appears that the production of antibodies in RA involves the generation of IgG against CEmodified DNA through oxidative induced DNA damage. Results imply that oxidative damage to DNA alter it immunogenicity leading to the induction and elevated levels of RA antibodies. RA is a chronic, systemic, inflammatory condition that is characterized by increased production of inflammatory cytokines as well as alteration in estrogen metabolites (CEs). Only by understanding the complex interaction between various estrogen metabolites and mechanism of autoantibodies production, we will able to generate therapeutic interventions that can be designed to suppress synovial inflammation in RA.

\section{Acknowledgements}

Authors like to thank Prof. Farhatullah for his valuable contribution to the editing of this manuscript.

\section{Author details}

Wahid Ali Khan ${ }^{1 *}$ and Mohd. Wajid Ali Khan²

*Address all correspondence to: wahidalikhan@rediffmail.com 
1 Department of Clinical Biochemistry, College of Medicine, King Khalid University , Kingdom of Saudi Arabia

2 Institute of Infection and Immunity, School of Medicine, Cardiff University, United Kingdom

\section{References}

[1] Belous, A. R., Hachey, D. L., Dawling, S., Roodi, N., \& Parl, F. F. (2007). Cytochrome p450 1B1-mediated estrogen metabolism results in estrogen-deoxyribonucleoside adduct formation. Cancer Res, 67, 812-817.

[2] Burrage, P. S., Mix, K. S., \& Brinckerhoff, C. E. (2006). Matrix metalloproteinases: role in arthritis. Front Biosci, 1, 529-543.

[3] Camussi, G., \& Lupia, E. (1998). The future role of anti-tumors necrosis factors (TNF) products in the treatment of rheumatoid arthritis. Drugs, 55, 613-620.

[4] Castagnetta, L. M. A., Granata, O. M., Traina, A., Ravazzolo, B., Amoroso, M., Miele, M., Bellavia, V., Agostara, B., \& Carruba, G. (2002). Tissue content of hydroxyestrogens in relation to survival of breast cancer patients. Clin Cancer Res, 8, 3146-3155.

[5] Castnegnetta, L., Carruba, G., Granata, O. M., Stefano, R., Miele, M., Schmidt, M., Cutolo, M., \& Straub, R. H. (2003). Increase estrogen formation and estrogen to androgen ratio in the SF of patients with rheumatoid arthritis. J Rheumatoid, 30, 2597-2605.

[6] Castnegnetta, L., Cutolo, M., Granata, O., Falco, M., Bellavia, V., \& Carruba, G. (1999). Endocrine end points in rheumatoid arthritis. Ann NY Acad Sci, 876, 180-192.

[7] Cavalieri, E., Frenkel, K., Liehr, J. G., Rogan, E., \& Roy, D. (2000). Estrogens as endogenous genotoxic agents-DNA adducts and mutations. J Natl Cancer Inst Monogr, 27, 75-93.

[8] Chen, Z. H., Na, H. K., Hurh, Y. J., \& Surh, Y. J. (2005). Hydroxyestradiol induces oxidative stress and apoptosis in human mammary epithelial cells: possible protection by NF- $\kappa B$ and ERK/MAPK. Toxicol Appl Pharmacol , 208, 46-56.

[9] Cooke, M. S., Mistry, N., Wood, C., Herbert, K. E., \& Lunec, J. (1997). Immunogenicity of DNA damaged by reactive oxygen species-implications for anti-DNA antibodies in lupus. Free Radic Biol Med, 22, 151-9.

[10] Cutolo, M., Capellino, S., Sulli, A., Serioli, B., Secchi, M. E., Villaggio, B., \& Straub, R. H. (2006). Estrogens and autoimmune diseases. Ann N Y Acad Sci, 1089, 538-547.

[11] Cutolo, M., \& Straub, H. (2012). Estrogen metabolism and autoimmunity. Autoimmun Rev, 11, 460-464. 
[12] Cutolo, M., Sulli, A., Seriolo, B., Accardo, S., \& Masi, A. T. (1995). Estrogen, the immune responses and autoimmunity. Clin Exp Rheumatol, 13, 217-226.

[13] Cutolo, M., Villaggio, B., Seriolo, B., Montagna, P., Capellino, S., Straub, R. H., \& Sulli, A. (2004). Synovial fluid estrogen in rheumatoid arthritis. Autoimmune Rev, 3, 193-198.

[14] Cutolo, M. (1998). The role of the hypothalamus-pituitary-adrenocortical and-gonadal axis in rheumatoid arthritis. Clin Exp Rheumatol, 16, 3-6.

[15] Cutolo, M. (2004). Estrogen metabolites: increasing evidence for their role in rheumatoid arthritis and systemic lupus erythematosus. J Rheumatol, 31, 419-421.

[16] Cutolo, M. (2010). Hormone therapy in rheumatoid diseases. Curr Opin Rheumatol, $22,257-263$.

[17] Deng, H. W., \& Shen, H. (2002). Current topics in human genetics: studies in complex diseases. In: Long JR. Genes in estrogen Metabolism Pathway and Breast Cancer, Long JR, World Scientific Publishing. 759-780.

[18] Devanesan, P., Santen, R. J., Bocchinfuso, W. P., Korach, K. S., Rogan, E. G., \& Cavalieri, E. (2001). Catecholestrogen metabolites and conjugates in mammary tumors and hyperplastic tissue from estrogen receptor- $\alpha$ knock-out (ERKO)/Wnt-1 mice: Implications for initiation of mammary tumors. Carcinogenesis, 22, 1573-1576.

[19] Doria, A., Ghirardello, A., Iaccarino, L., Zampieri, S., Punzi, L., Tarricone, E., Ruffatti, A., Sulli, A., Sarzi-Puttini, P. C., Gambari, P. F., \& Cutolo, M. (2004). Pregnancy cytokines and disease activity in systemic lupus erythematosus. Arthritis Rheum, 51, 989-995.

[20] Dubey, R. K., \& Jackson, E. K. (2001). Cardiovascular protective effect of $17 \beta$-estradiol metabolites. J Appl Physio, 91, 1868-1883.

[21] Embrechts, J., Lemiere, F., Van Dongen, W., Esmans, E. L., Buytaert, P., Van Marck, E., Kockx, M., \& Makar, A. (2003). Detection of estrogen DNA-adducts in human breast tumor tissue and healthy tissue by combined nano LC-nano ES tandem mass spectroscopy. J Am Soc Mass Spectrom, 14, 482-491.

[22] Firestein, G. S. (2003). Evolving concepts of rheumatoid arthritis. Nature, 423, 356-361.

[23] Frenkel, K., Karkoszka, J., Glassman, T., Dublin, N., Toniolo, P., Taioli, E., Mooney, L. A., \& Kato, I. (1998). Serum autoantibodies recognizing 5-hydroxymethyl-2'-deoxyuridine, an oxidized DNA base, as biomarkers of cancer risk in women. Cancer Epidemiol Biomarkers Prev, 7, 49-57.

[24] Gaub, M., Bellard, M., Sheuer, I., Chambon, P., \& Sassone-Carsip, . (1990). Activation of the ovalbumin gene by the estrogen receptor involves the Fos-Jun complex. Cell, $63,1267-1276$.

[25] George, J., \& Shoenfeld, Y. (1996). Natural autoantibodies. In: Autoantibodies, Peter JB, Shoenfeld Y, editors, Elsevier Scientifi, Amsterdam. 539. 
[26] Granner, D. K. (1996). Hormones of the adrenal cortex. In: Harper's Biochemistry, 24th edn, Murray RK, Granner DK, Mayes PA, Rodwell VW, Appleton E Lange, Stanford., 547-591.

[27] Grossman, J., \& Brahn, M. (1997). Rheumatoid arthritis: current clinical and research directions. J Womens Health, 6, 627-638.

[28] Hammond, D. K., Zhu, B. T., Wang, M. Y., Ricci, M. J., \& Liehr, J. G. (1997). Cytochrome p450 metabolism of estradiol in hamster liver and kidney. Toxicol Appl Pharmacol, 145, 54-60.

[29] Hoffman, A. R., Paul, S. M., \& Axelrod, J. (1980). The enzymatic formation of catecholestrogen from 2 methoxyestrogens by rat liver microsomes. Endocrinol, 107, 1192-1197.

[30] Inversion, S. L., Shen, L., Anlor, N., \& Bolton, J. L. (1996). Bioactivation of estrone and its catechol metabolites to quinoid-glutathione conjugates in rat liver microsomes. Chem Res Toxicol, 9, 492-499.

[31] Ito, A., Bebo, B. F., Jr, Mateguk, A., Zamora, A., Silverman, M., Fyfe-Johnson, A. ., \& Offer, H. (2001). Estrogen treatment down-regulates TNF-alpha production and reduces the severity of experimental autoimmune encephalomyelitis in cytokine knockout mice. J. Immunol, 167, 542-552.

[32] Janele, D., Lang, T., Capellino, S., Cutolo, M., Dasilva, J. A. P., \& Straub, R. H. (2006). Effect of testosterone, $17 \beta$-estradiol and down stream estrogen on cytokine secretion from leukocytes in the presence and absence of cortisol. Ann N Y Acad Sci, 1069, 168-182.

[33] Kanda, N., Tsuchida, T., \& Tamaki, K. (1999). Estrogen enhancement of anti-doublestranded DNA antibody and immunoglobin G production in peripheral blood mononuclear cells from patients with systemic lupus erythematosus. Arthritis Rheum, 42, 328-337.

[34] Kavanaugh, A., \& Lipsky, P. E. (1996). Rheumatoid arthritis. In: Chemical immunology: principles and practice, Rich RR, Schwartz BD, Fleisher TA, Shearer WT, Strober W, Mosby-Year Book, ST Louis, MO., 1093.

[35] Khalkhali-Ellis, Z., Seftor, E. A., Nieva, D. R., Handa, R. J., Price, R. H., Price, R. H., Jr, Kirschmann, D. A., Baragi, V. M., Sharma, R. V., Bhalla, R. C., Moore, T. L., \& Hendrix, M. J. (2000). Estrogen and progesterone regulation of human fibroblast-like synoviocyte function in vitro: implication in rheumatoid arthritis. J Rheumatol, 27, 1622-1631.

[36] Khan, W. A., Alam, K., \& Moinuddin, . (2007). Catechol-estrogen modified DNA: a better antigen for cancer autoantibody. Arch Biochem Biophys, 465, 293-300.

[37] Khan, W. A., Habib, S., Khan, M. W. A., Alam, K., \& Moinuddin. (2008). Enhanced binding of circulating SLE autoantibodies to catecholestrogen-copper-modified DNA. Mol Cell Biochem, 315, 143-150. 
[38] Khan, W. A., \& Moinuddin, Assiri. A. S. (2011). Immunochemical studies on catecholestrogen modified plasmid: possible role in rheumatoid arthritis. J Clin Immunol, 31, 22-29.

[39] Khan, W. A., Moinuddin, Khan. M. W. A., \& Chabbra, H. S. (2009). Catecholestrogen: possible role in systemic lupus erythematosus. Rheumatol (Oxford), 48, 1345-1351.

[40] Khan, W. A., \& Moinuddin, . (2006). Binding characteristics of SLE anti-DNA autoantibodies to catecholestrogen-modified DNA. Scand J Immunol, 64, 677-683.

[41] Knobil, E., \& Neil, J. D. (2006). Knobil and Neill's physiology of reproduction. 3rd Ed.Gulf Professional Publishing, , 2352-2376.

[42] Komi, J., \& Lassila, O. (2000). Nonsteroidal anti-estrogens inhibit the functional differentiation of human monocyte-derived dendritic cells. Blood, 95, 2875-2882.

[43] Lahita, R. G., Bradlow, H. L., Kunkel, H. G., \& Fishman, J. (1979). Alteration of estrogen metabolism in systemic lupus erythematosus. Arthritis Rheum, 22, 1195-1198.

[44] Lahita, R. G. (1996). The connective tissue diseases and the over all influence of gender. Int J Fertil Menopausal Stud, 41, 156-160.

[45] Le Bail, J., Liagre, B., Vergne, P., Bartin, P., Beneytout, J., \& Habrioux, G. (2001). Aromatase in synovial cells from postmenopausal women. Steroids, 66, 749-753.

[46] Li, J. J., \& Li, S. A. (1987). Estrogen carcinogenesis in Syrian hamster tissue: role of metabolism. Fed Proc, 46, 1858-1863.

[47] Li, K. M., Todovic, R., Devansen, P., Higginbotham, S., Kofeler, H., Ramanathan, R., Gross, M. L., Rogan, E. G., \& Cavalieri, E. (2004). Metabolism and DNA binding studies of 4-hydroxyestradiol and estradiol-3,4-quinone invitro and in female ACI rat mammary gland invivo. Carciongenesis , 25, 289-297.

[48] Liehr, J. G., \& Racci, M. J. (1996). 4-Hydroxylation of estrogens as marker of human mammary tumors. Proc Natl Acad Sci USA, 93, 3294-3296.

[49] Lin, P. H., Nakamura, J., Yamaguchi, S., Asakura, S., \& Swenberg, J. A. (2003). Aldehydic DNA lesions induced by catecholestrogens in calf thymus DNA. Carcinogenesis, 24, 1133-1141.

[50] Majithia, V., \& Geracia, S. A. (2007). Rheumatid arthritis: diagnosis and management. Am J Med, 120, 936-939.

[51] Markushin, Y., Zhong, W., Cavalieri, E. L., Rogan, E. G., Small, G. J., Yeung, E. S., \& Jankowiak, R. (2003). Spectral characterization of catechol estrogen quinone (CEQ)derived DNA- adducts and their identification in human breast tissue extract. Chem Res Toxicol, 16, 1107-1117.

[52] Mor, G., Wei, Y., Santen, R. J., Gutierrez, L., Eliza, M., Lev, B., Harada, N., Wang, J., Lysiak, J., Diano, S., \& Naftolin, F. (1998). Macrophages, estrogen and the microenvironment of breast cancer. J Steroid Biochem Mol Biol, 67, 403-411. 
[53] Parhan, P. (2000). The Immune System. Elsevier Science Inc, New York, NY.

[54] Paul, S. M., \& Axelrod, J. (1977). Catecholestrogens: presence in brain and endocrine tissues. Science, 197, 657-659.

[55] Purohit, A., Ghilchic, M. W., \& Duncan, L. (1995). Aromatase activity and interleukin-6 production by normal and malignant breast tissues. J Clin Endocrinol Metab, 80, 3052-3058.

[56] Roftogianis, R., Creveling, C., Weinshilboum, R., \& Weisz, J. (2000). Estrogen metabolism by conjugation. J Natl Cancer Inst Monogr, 27, 113-124.

[57] Rogan, E. G., Badawi, A. F., Devanesan, P. D., Meza, J. L., Edney, J. A., West, W. W., Higginbotham, S. M., \& Cavalieri, E. L. (2003). Relative imbalances in estrogen metabolism and conjugation in breast tissues of women with carcinoma: Potential biomarkers of susceptibility to cancer. Carcinogenesis, 24, 697-702.

[58] Saeed, M., Rogan, E., Fernandez, S. V., Sheriff, F., Russo, J., \& Cavalieri, E. (2007). Formation of depurinating N3 Adenine and N7 Guanine adducts by MCF-10 F cells cultured in the presence of 4-hydroxyestradiol. Int J Cancer, 120, 1821-1824.

[59] Safe, S. H. (1998). Interactions between hormones and chemicals in breast cancer. Annu Rev Pharmacol Toxicol, 38, 121-158.

[60] Schmidt, M., Hartung, R., Capellino, S., Cutolo, M., Pfeifer-Leeg, A., \& Straub, R. H. (2009). Estrone/17 $\beta$-estradiol conversion to and tumor necrosis factor inhibition by, estrogen metabolites in synovial cells of patients with rheumatoid arthritis and patients with osteoarthritis. Arthritis Rheum, 60, 2913-2922.

[61] Schneider, J., Huh, M. M., Bradlow, H. L., \& Fishman, J. (1984). Antiestrogen action of 2-hydroxyestrone of MCF-7 human breast cancer cells. J Biol Chem, 259, 4840-4845.

[62] Schutze, N., Vollmer, G., \& Knuppen, R. (1994). Catecholestrogens are agonists of estrogen receptor dependent gene expression in MCF-7 cells. J Steroid Biochem Mol Biol, $48,453-461$.

[63] Scott, D. L., \& Kingsley, G. H. (2006). Tumor necrosis factor inhibitors for rheumatoid arthritis. N Engl J Med, 355, 704-712.

[64] Steiner, G., \& Smolen, J. (2002). Autoantibodies in rheumatoid arthritis and clinical significance. Arthritis Res , 4, S 1-S5.

[65] Straub, R., \& Cutolo, M. (2001). Impact of the hypothalamic pituitary-adrenal/gonadal oxes and the peripheral nervous system in rheumatoid arthritis: a systemic pathogenic view point. Arthritis Rheum, 44, 493-507.

[66] Straub, R. H. (2007). The complex role of estrogens in inflammation. Endocr Rev, 28, 521-574.

[67] Strauss, J. F., \& Barbieri, R. L. (2009). In: Yen and Jaffe's reproductive endocrinology: physiology, pathophysiology and clinical management. $6^{\text {th }}$ Ed, Elsevier Health Science., 98. 
[68] Weidler, C., Harle, P., Schedel, J., Schmidt, M., Scholmuich, J., \& Straub, R. H. (2004). Patients with rheumatoid arthritis and systemic lupus erythematosus have increased renal excretion of mitogenic estrogens in relation to endogenous antiestrogens. $J$ Rheumatol, 31, 489-494.

[69] Yue, W., Santen, R. J., Wang, J. P., Li, Y., Verderame, M. F., Bocchinfuso, W. P., Korach, K. S., Devanesan, P., Todorovic, R., Rogan, E. G., \& Cavalieri, E. L. (2003). Genotoxic metabolites of estradiol in breast: potential mechanism of estradiol induced carcinogenesis. J Steroid Biochem Mol Biol, 86, 477-486.

[70] Zhu, B. T., \& Conney, A. H. (1998). Functional role of estrogen metabolism in target cells: review and perspectives. Carcinogenesis, 19, 1-27.

[71] Zutshi, D. W., Reading, C. A., Epstein, W. V., Anseel, B. M., \& Holborow, E. J. (1969). FII haemagglutination test for serum antigammaglobulin factors in arthritis sero-positive and sero-negative by other tests. Ann Rheum Dis, 28, 289-299. 